\title{
Photon locking
}

\author{
E. T. Sleva, I. M. Xavier, Jr., ${ }^{*}$ and A. H. Zewail \\ Arthur Amos Noyes Laboratory of Chemical Physics, California Institute of Technology, Pasadena, \\ California 91125
}

Received September 30, 1985; accepted November 19, 1985

\begin{abstract}
A novel observation of photon locking-the optical analog of spin locking-is reported, demonstrating the applicability of phase-coherent pulse sequences. The experiments are reported for the optical transition of iodine gas at $589.7 \mathrm{~nm}$ using the pulse sequence $X Y X-X Y \bar{X}$. Locking decay rates are presented as a function of pressure and compared with optical dephasing (echo-decay) rates.
\end{abstract}

\section{INTRODUCTION}

Modulation of a cw laser by a traveling-wave acousto-optic modulator has recently been utilized to implement sequences of phase-coherent optical pulses. ${ }^{1}$ By controlling the relative phases of the rf pulses applied to the crystal, one can control the phases of the light pulses that are created by the acoustic grating. That is, the phase of an rf pulse is effectively transferred to the corresponding light pulse. The execution of a sequence of phase-coherent light pulses is thus reduced to generating the corresponding sequence in the rf domain. We describe here the first reported use of this technique to effect the optical analog of spin locking.

In both the spin-locking experiment and its optical analog, a $\pi / 2$ pulse along the $x$ axis of the rotating frame is followed by the application of an intense field along the $y$ axis. ${ }^{2}$ In our experiment, the first pulse serves to prepare a narrowband coherence within the inhomogeneously broadened linewidth of gaseous iodine. The intense $y$-axis field (phase shifted by $90^{\circ}$ from the preparation pulse) serves to prevent dephasing of the initially prepared coherence by frequency offsets (which may arise from inhomogeneous broadening or velocity-changing collisions). $T_{1 \rho}$, the decay time of the coherence under these conditions, is obtained by continuously varying the duration of the locking field pulse. To detect the locked coherence, we add a pulse to the usual twopulse locking sequence; this third pulse serves to convert the locked coherence to an excited-state population enhancement or depletion. ${ }^{3,4}$ The coherent signal of interest is in this manner detected by observing only (incoherent) fluorescence, using the following sequence:

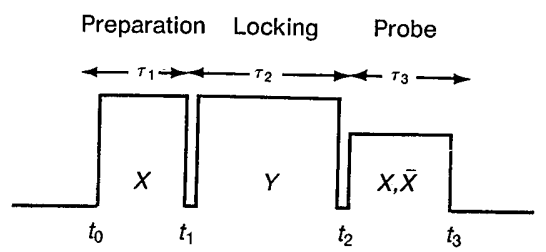

We have measured as a function of pressure optical $T_{1 \rho}$ for the $X^{1} \Sigma_{g}{ }^{+} \rightarrow B^{3} \Pi_{O^{+}}$transition of iodine at $589.7 \mathrm{~nm}$. Over the range of pressures studied, $T_{1 \rho} \approx T_{2}$. To complement our experimental studies, computer simulations using the optical Bloch equations were performed in which signal was integrated over both the Doppler linewidth and the transverse-laser-beam intensity profile.

\section{EXPERIMENT}

An argon-ion laser was used to pump a Spectra-Physics Model 380A single-mode ring dye laser. Laser mode structure was monitored with a scanning Fabry-Perot interferometer, and the wavelength was determined with a JarrellAsh monochromator. Continuous-wave radiation $(\approx 300$ $\mathrm{mW}$ ) at $589.7 \mathrm{~nm}$ from the ring dye laser was focused into a Harris Model 190 acousto-optic modulator. The modulator was driven by rf $(460-\mathrm{MHz})$ pulses. The $\mathrm{rf}$ source is split into the three phase components of interest, each of which is regulated by an $\mathrm{rf}$ switch before recombination with the others. The relative phases were set with a Hewlett-Packard Model 8405A vector voltmeter. Optical pulses were detected after passage through the sample cell by a fast photodiode and were displayed on an oscilloscope throughout the experiment. A photomultiplier tube was used to detect the fluorescence emitted in a direction perpendicular to the direction of laser-beam propagation. In this scheme, only incoherent emission is detected. The signal was preamplified and processed by an EG\&G Model 165 gated integrator and a Model 162 boxcar averager. The integrator gate was set to follow the probe pulse $(X$ or $\bar{X})$; the baseline sampling feature of the integrator was used to subtract that signal obtained with the $\bar{X}$ pulse (i.e., that which is $180^{\circ}$ out of phase with the excitation pulse) from that obtained with the $X$ pulse.

The sample cell consists of a glass bulb with Brewster windows and a Teflon stopcock. Before sample preparation, the cell was baked out with a heating tape while attached to a vacuum manifold until a vacuum of approximately $1 \times 10^{-6}$ Torr was achieved. Iodine was then introduced by distillation at $0^{\circ} \mathrm{C}$ into a side arm of the sample cell. This side arm was also used during the experiments to immerse the iodine crystals in various low-temperature baths in order to control the vapor pressure. The dependence of iodine vapor presure on temperature is given by an equation of Giauque. ${ }^{5}$

\section{THEORY}

The optical Bloch equations were assumed as a model for the behavior of $\mathrm{I}_{2}$ under the conditions of our experiment. The pulse sequence in which the probe pulse is in phase with the preparation pulse is referred to as $X Y X$; the sequence with the phase-shifted probe, as $X Y \bar{X}$. 
Before introducing the effect of severe inhomogeneous broadening, it is convenient to consider the case in which the Doppler width is much smaller than the Rabi frequency. Use of the Feynman-Vernon-Hellwarth formalism ${ }^{6}$ will facilitate these considerations. The locking sequence consists of a $\pi / 2$ pulse applied along the $x$ axis of the rotating frame, followed by an intense locking field applied along the $y$ axis of the rotating frame. The locking field serves to suppress inhomogeneous dephasing; observing the temporal decay of the locked coherence should accordingly give the homogeneous linewidth. The coherence may be detected by applying a third pulse; subtraction of the signal obtained with the $X Y \bar{X}$ sequence from that obtained with the $X Y X$ yields the signal of interest.

Now consider the case in which the Doppler width is greater than the Rabi frequency. In this circumstance there are important off-resonance and flip-angle effects. At early times, the well-known composite-pulse effect ${ }^{7}$ will completely mask the signal of interest. At zero time the probe pulse follows the preparation pulse immediately (there is no $Y$ pulse at zero time). The signal for $X X$ will be the fluorescence obtained by applying a pulse of duration $\tau_{1}+\tau_{3}$, whereas the signal for $X \bar{X}$ will be that for a single pulse of the same duration in which the phase has been shifted by $180^{\circ}$ at time $t_{1}$. Because of the phase reversal in the $X \bar{X}$, it has a greater bandwidth and gives rise to a larger signal than the $X X$. This effect is predicted by computer simulations and has been observed experimentally. ${ }^{1}$ Simulations were performed to determine the relationship between our signal and the locked coherence, which, of course, is the quantity of interest.

During a given pulse, the optical Bloch equations constitute a nonhomogeneous linear system with constant coefficients. Consider a particular frequency offset and electricfield intensity. One calculates signal intensity for the given values of these parameters and subsequently integrates over $\Delta$ for the Doppler profile and $\omega_{R}$ (the Rabi frequency) for the transverse laser profile:

$$
\dot{\mathbf{x}}=\mathbf{A r}+\mathbf{g},
$$

where $\mathbf{A}$ is a constant matrix throughout the duration of a particular pulse. For the pulse sequence of interest,

and

$$
\mathbf{A}=\left(\begin{array}{ccc}
-1 / T_{1 \rho} & \Delta & -\omega_{R y} \\
-\Delta & -1 / T_{1 \rho} & \omega_{R_{x}} \\
\omega_{R y} & -\omega_{R_{x}} & -1 / T_{1}
\end{array}\right)
$$

$$
\mathbf{g}=\left(\begin{array}{c}
0 \\
0 \\
-1 / T_{1}
\end{array}\right)
$$

$\mathbf{r}$ is made up of linear combinations of elements from the two-level density matrix. $r_{1}$ and $r_{2}$ correspond to the combination of off-diagonal elements that give rise to polarization along the $x$ and $y$ axes, respectively, of the rotating frame. $r_{3}$ is proportional to $\rho_{22}-\rho_{11}$, the population difference. $T_{1 \rho}$ is the transverse and $T_{1}$ the longitudinal decay time. $\Delta$ is the frequency offset, while $\omega_{R_{x}}$ and $\omega_{R_{y}}$ are the Rabi frequencies of pulses along the $x$ and $y$ axes, respectively. For an $x$ pulse, $\omega_{R y}=0$; similarly, for a $y$ pulse, $\omega_{R_{x}}=0$. Equation (1) is solved by diagonalization of $\mathbf{A}$, which decouples the three

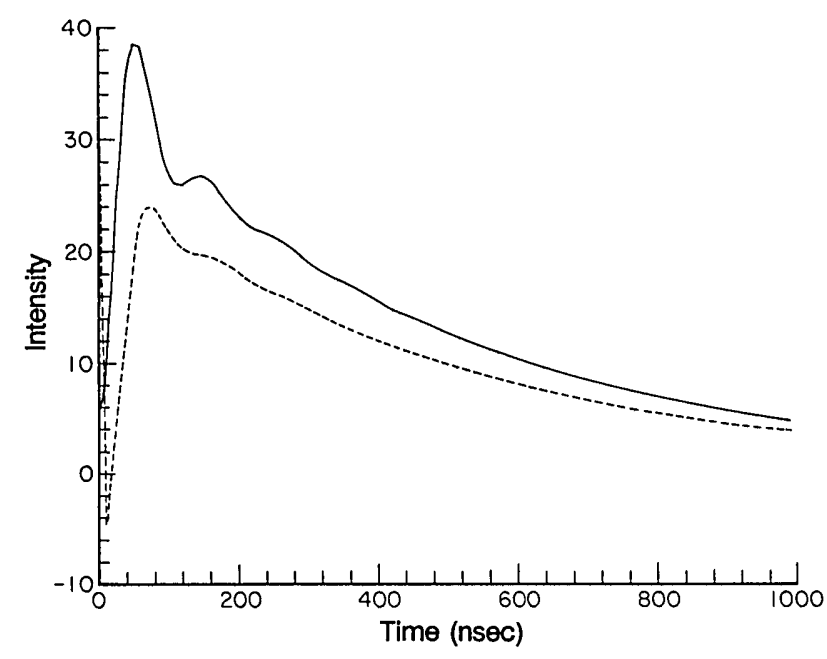

Fig. 1. Computer simulation of locking decay signals using the optical Bloch equations. The dashed line represents the conventional polarization signal $r_{2}$ (see text), the solid line the signal that is expected with our probe pulse technique, which converts the polarization to fluorescence $\left(r_{3}\right)$. Here $\tau_{1}=\tau_{3}=50 \mathrm{nsec}, \omega_{R 1}(\rho=0)=\omega_{R 3}$ $(\rho=0)=0.03 \mathrm{nsec}^{-1}$, and $\omega_{R} 2(\rho=0)=0.06 \mathrm{nsec}^{-1}$.

equations, thereby reducing the problem to that of solving three independent nonhomogeneous linear equations with constant coefficients. Let $\mathbf{r}=\mathbf{T R}$. Then

$$
\dot{\mathbf{R}}=\mathbf{D R}+\mathbf{h} \text {, }
$$

where $\mathbf{D}=\mathbf{T}^{-1} \mathbf{A T}$ is diagonal and $\mathbf{h}=\mathbf{T}^{-1} \mathbf{g}$. The uncoupled equations are solved, back transformation with $\mathbf{T}$ recovers $\mathbf{r}$, and the solution at the end of a given pulse is used as the initial condition for the succeeding pulse. During the excitation and probe pulses relaxation is neglected (i.e., $T_{1}$ and $T_{1 \rho}$ are assumed to be infinite during these pulses because the pulses are much shorter than $T_{1}$ or $T_{1 \rho}$ ). Thus, after the first pulse,

$$
\mathbf{r}\left(t_{1}\right)=\left(\begin{array}{c}
\left(\omega_{R 1} \Delta / \Omega_{1}^{2}\right)\left[1-\cos \left(\Omega_{1} t_{1}\right)\right] \\
\left(\omega_{R 1} / \Omega_{1}\right) \sin \left(\Omega_{1} t_{1}\right) \\
-\left[\Delta^{2}+\omega_{R 1}{ }^{2} \cos \left(\Omega_{1} t_{1}\right)\right] / \Omega_{1}{ }^{2}
\end{array}\right),
$$

where $\omega_{R 1}$ is the Rabi frequency of the first pulse and $\Omega_{1}=$ $\left(\Delta^{2}+\omega_{R 1}{ }^{2}\right)^{1 / 2}$. For a particular $\omega_{R}$ and $\Delta, \mathbf{r}\left(t_{3}\right)$ must be calculated for a probe pulse that is in phase $(X)$ and out of phase $(\bar{X})$ with the excitation pulse. The third component of the former minus that of the latter yields the signal of interest on integration over the Doppler and transverse laser-intensity profiles. Denoting the phase of the probe pulse by $\phi$, this is

$$
I=\int_{\Delta, \rho} \rho\left[r_{3}\left(t_{3}, \Delta, \rho, \phi=X\right)-r_{3}\left(t_{3}, \Delta, \rho, \phi=\bar{X}\right)\right] \mathrm{d} \Delta \mathrm{d} \rho,
$$

where the distribution of Rabi frequencies is given by $\omega_{R}=$ $\omega_{R 0} \exp \left(-\rho^{2}\right), \rho$ being the radial coordinate for the (assumed Gaussian) transverse beam profile and $\omega_{R 0}$ the Rabi frequency at the center of the laser beam. In this treatment we do not consider deviations from the Bloch equations to be due to dependence of $T_{2}$ on field strength. ${ }^{8}$ As shown later, the locking decay time $T_{1 \rho}$ is roughly equal to $T_{2}$, and $T_{2}$ shows a 
well-behaved pressure dependence that its in agreement with previous work by Brewer's group and ours. $3,9,10$

An FPS-164 array processor was used to average over the inhomogeneous linewidth and the transverse beam profile. Figure 1 shows for comparison the predicted decay for the observed signal along with that for the polarization. The signal yields the decay of interest once the composite pulse effect has subsided.

\section{RESULTS AND DISCUSSION}

Shown in Fig. 2 are signals for the locking experiment, an optical free-induction decay, and a photon echo; these data
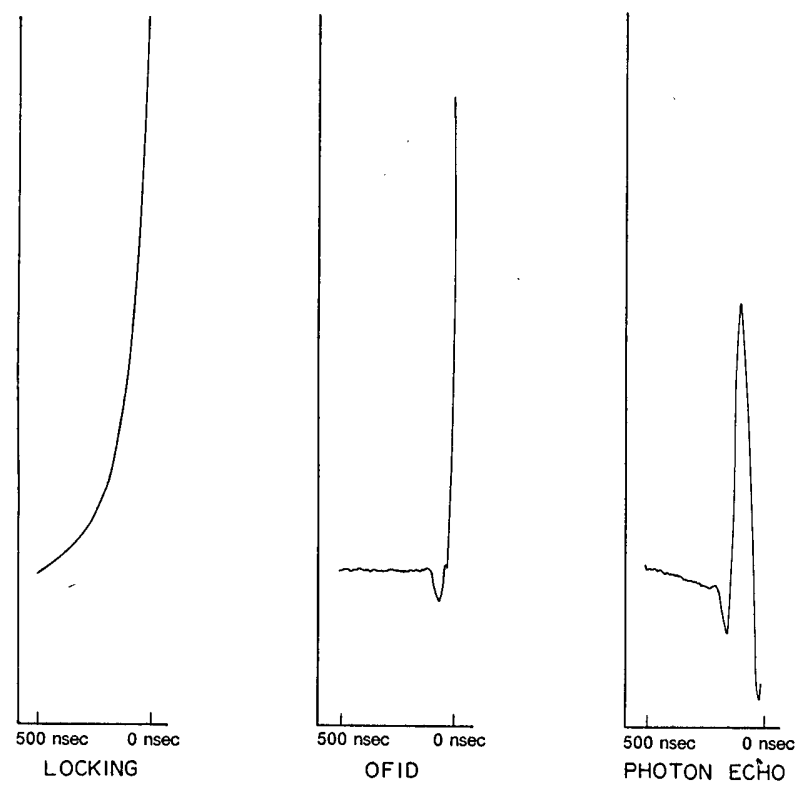

Fig. 2. Locking, optical free-induction decay (OFID), and photon echo signals using the probe pulse method. The $I_{2}$ pressure is 30 mTorr. The sequences used are $X Y X(\bar{X})$ for locking, $X X X(\bar{X})$ for the echo, and $X X(\bar{X})$ for the OFID.

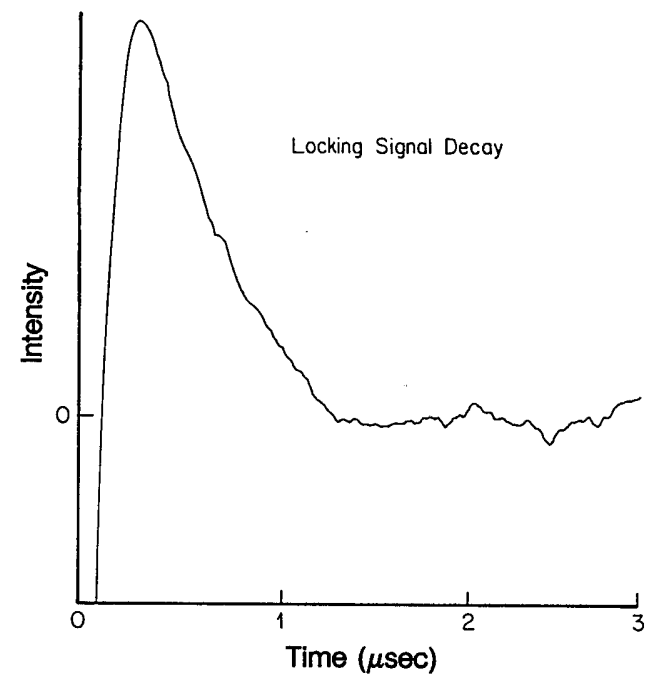

Fig. 3. Locking signal decay $\tau_{2}$ is scanned continuously. At all times, the third pulse follows the $y$ pulse immediately. The laser power during the preparation and locking pulses was $100 \mathrm{~mW}$; during the probe pulse, $20 \mathrm{~mW}$. The preparation pulse duration was $50 \mathrm{nsec}$; the probe $150 \mathrm{nsec}$.

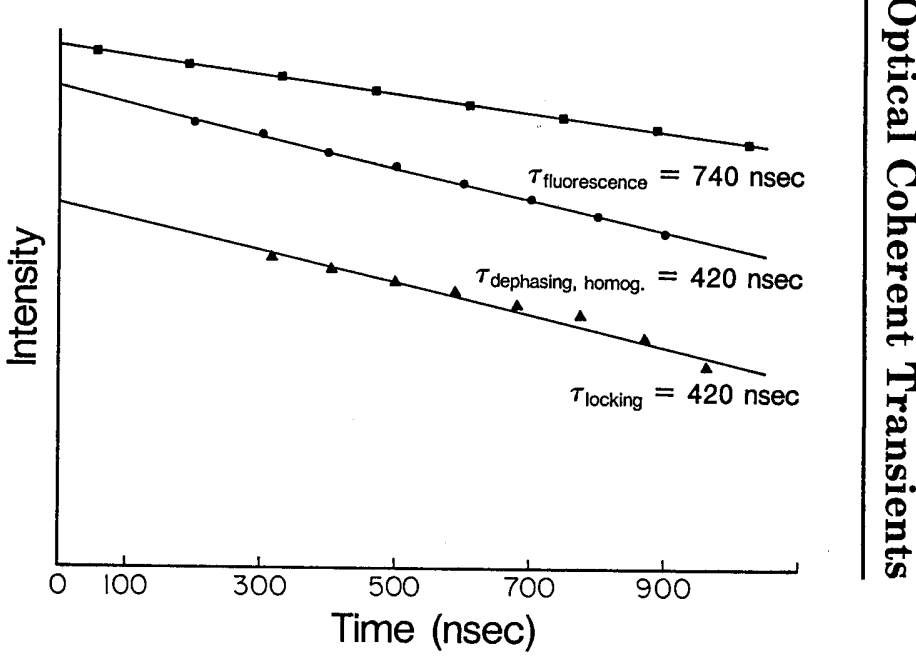

Fig. 4. For comparison: fluorescence, echo, and locking decays at 30 mTorr. Note that $T_{2} \approx T_{1 \rho}$.

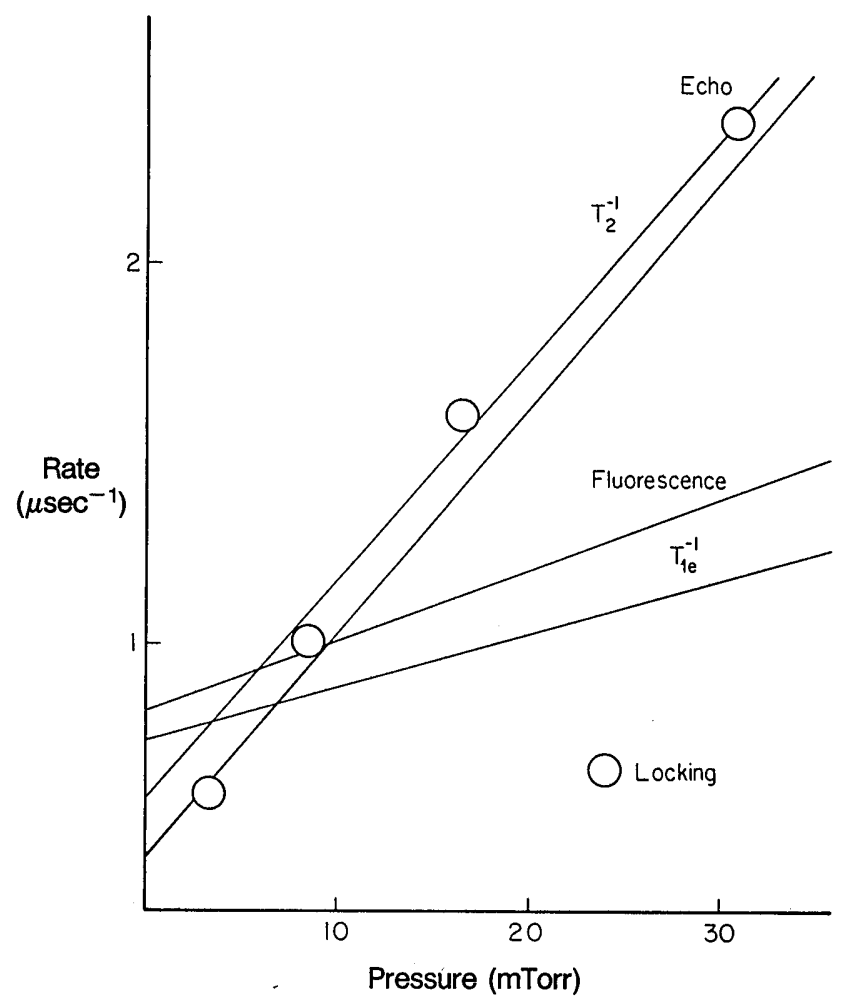

Fig. 5. $\quad T_{1}, T_{2}$, and $T_{1 \rho}$ for $\mathrm{I}_{2}$ at $589.7 \mathrm{~nm}$ as a function of pressure. $T_{1 \rho} \approx T_{2}$. The circles are the measured locking decays $\left(T_{1 \rho}\right)$, and the solid lines are the measured $T_{2}$ and $T_{1}$ decays (with their error bars) obtained here and in previous work (see Ref. 10).

were all taken using the $X, \bar{X}$ probe pulse technique. The pressure of $\mathrm{I}_{2}$ gas was maintained at $30 \mathrm{~m}$ Torr. Figure 3 shows a decay of the locking signal at this pressure. This decay resembles the simulation of Fig. 1, which was obtained by using Eq. (6).

The purpose of locking is to prevent dephasing due to frequency offsets, such as those encountered in inhomogeneously broadened systems or caused by velocity-changing collisions. Optical inhibition of dephasing may be effected only if the Rabi frequency exceeds the magnitude of the relevant frequency offsets; this condition is a well-known 
criterion for the usefulness of spin locking in magnetic resonance. The groups of Hartmann and Mossberg have demonstrated that radiation may be used to inhibit certain collisional and inhomogeneous decay mechanisms in photonecho experiments. ${ }^{11,12}$ In these experiments, however, the relative phases of the pulses were not prescribed. Our experiment requires that the locking field be phase shifted by $90^{\circ}$ from the excitation pulse; this pulse sequence is the direct optical analog of spin locking, and the locking decay yields optical $T_{1 \rho}$ directly.

Figure 4 shows a log fit of locking, echo, and fluorescence decays at $30 \mathrm{mTorr}$ for comparison; Fig. 5 is a plot of $T_{1 \rho}$ and $T_{2}$ against pressure. The data indicate that $T_{1 \rho}$ is roughly the same as $T_{2}$ over the range of pressures studied. ${ }^{13}$ These results indicate that for iodine in the pressure range studied, coherent radiation (the $y$ pulse in our experiment) suppresses dephasing that is due to inhomogeneous broadening and velocity-changing collisions when the Rabi frequency is larger than the frequency shifts. A collision may, of course, induce both phase interruption and a change in velocity. In the iodine case, both types of collision have been considered for explaining the $T_{2}$ decay. $3,9,10,14$ In a separate publication we will discuss these mechanisms when all pressure-dependence measurements are completed and studies of the effect of flip angle and $y$ pulse intensity are made.

\section{CONCLUSIONS}

In conclusion, we have reported the first use to our knowledge of phase-coherent pulses to effect the optical analog of spin locking. We intend to exploit the potential that this pulse sequence has for the study of solids and isolated molecules.

\section{ACKNOWLEDGMENTS}

It is a pleasure to acknowledge stimulating discussions with W. S. Warren and a helpful discussion with R. G. Brewer. This research was funded by the National Science Founda- tion (DMR-8521191). I. M. Xavier, Jr., acknowledges a graduate fellowship from CAPES and UFPE (Brazil).

* On leave from Departamento de Quimica Fundamental, Universidade Federal de Pernambuco, 50000, Recife, Pernambuco, Brazil.

Note added in proof: Recently we observed photon locking in iodine gas using echo detection. A four-phase sequence $[X Y X X(\vec{X})]$ was used, and two echoes (for the locked and unlocked coherence) were observed. Details of this other work and related to isolated molecules can be found in Ref. 15.

\section{REFERENCES AND NOTES}

1. W. S. Warren and A. H. Zewail, J. Chem. Phys. 75, 5956 (1981); 78,2279 (1983).

2. A. G. Redfield, Phys. Rev. 98, 1787 (1955).

3. A. H. Zewail, T. E. Orlowski, K. E. Jones, and D. E. Godar, Chem. Phys. Lett. 48, 256 (1977); T. E. Orlowski, K. E. Jones, and A. H. Zewail, Chem. Phys. Lett. 54, 197 (1978).

4. T. E. Orlowski and A. H. Zewail, J. Chem. Phys. 70, 1390 (1979).

5. W. F. Giauque, J. Am. Chem. Soc. 53, 507 (1931).

6. R. P. Feynman, F. L. Vernon, and R. W. Hellwarth, J. Appl. Phys. 28, 49 (1957).

7. R. Freeman, S. P. Kempsell, and M. H. Levitt, J. Magn. Reson. 38, 453 (1980); M. H. Levitt and R. Freeman, J. Magn. Reson. 43, 502 (1981); M. H. Levitt, R. Freeman, and T. Frenkel, J. Magn. Reson. 47, 328 (1982).

8. R. G. DeVoe and R. G. Brewer, Phys. Rev. Lett. 50, 1269 (1983); A. Schenzle, M. Mitsunaga, R. G. DeVoe, and R. G. Brewer, Phys. Rev. A 30, 325 (1984).

9. R. G. Brewer and A. Z. Genack, Phys. Rev. Lett. 36, 959 (1979).

10. E. T. Sleva and A. H. Zewail, Chem. Phys. Lett. 110, 582 (1984).

11. N. W. Carlson, W. R. Babbit, Y. S. Bai, and T. W. Mossberg, Opt. Lett. 9, 232 (1984); P. F. Liao and S. R. Hartmann, Phys. Lett. 44A, 361 (1973).

12. A. G. Yodh, J. Golub, N. W. Carlson, and T. W. Mossberg, Phys. Rev. Lett. 53, 659 (1984).

13. At present, accurate error estimates are difficult to obtain because of single-mode instability, particularly at long times. However, the reproducibility of all pressure-dependence data was verified.

14. M. A. Banash, J. Gutow, and W. S. Warren, J. Lumin. 31-32, 855 (1984).

15. E. T. Sleva and A. H. Zewail, J. Phys. Chem. (to be published). 


\section{E. T. Sleva}

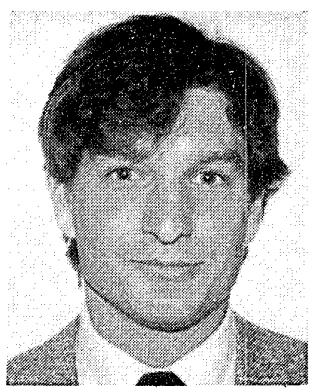

E. T. Sleva is a graduate student in chemical physics at the California Institute of Technology. Born in Cincinnati, Ohio, in 1959, he received bachelor's degrees in physics and chemistry from North Carolina State University in 1982. His research is concerned primarily with phase-coherent multiple-pulse optical spectroscopy. $\mathrm{He}$ is a member of the American Physical Society.

\section{M. Xavier, Jr.}

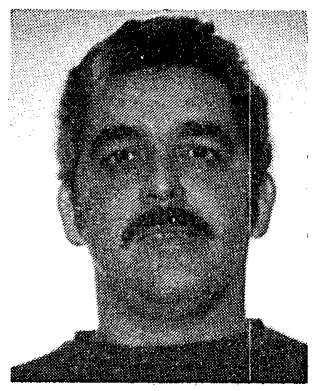

I. M. Xavier, Jr., is a graduate student in chemical physics at the California Institute of Technology. Born in Brazil, he received the bachelor's degree in chemical engineering and the master's degree in physics from the Federal University of Pernambuco. His research work includes coherent transients and state-tostate dynamics. $\mathrm{He}$ is a member of the Optical Society of America and the American Physical Society.

\section{A. H. Zewail}

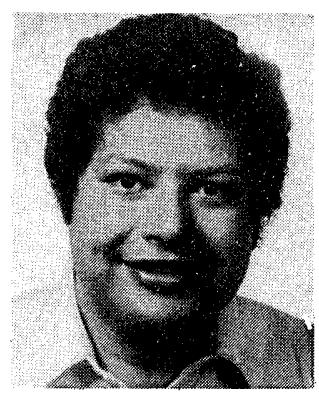

A. H. Zewail is professor of chemical physics at the California Institute of Technology. He was born in Egypt and received the bachelor's degree from $\mathrm{Al}$ exandria University. He obtained the $\mathrm{Ph} . \mathrm{D}$. degree in chemical physics from the University of Pennsylvania. After being a postdoctoral fellow at the University of California, Berkeley, he joined the faculty at the California Institute of Technology. He is a Fellow of the American Institute of Physics and has been an Alfred P. Sloan Fellow and Camille and Henry Dreyfus Teacher-Scholar. He is the author and coauthor of 150 publications and the editor of two books on laser spectroscopy and laser chemistry. 